\title{
Die Sulfochromite.
}

\author{
Von Max Gröger.
}

(Aus dem Laboratorium Prof. Zulkowsky's an der techn. Hochschule in Brïnn.)

(Vorgelegt in der Sitzung am 31. Marz 1881.)

In einer in den Sitzungsberichten der kais. Akademie der Wissenschaftten erschienenen Abhandlung ${ }^{1}$ sprach ich die Vermuthung ans, dass das Chromsulfid mit den Alkalisulfiden Doppelverbindungen einzugehen vermöge. Erhitzt man nämlich das am erwähnten Orte beschriebene Zinksulfochromit mit Natriumcarbonat in einem Kohlensäurestrome, ohne jedoch die Temperatur bis zum Schmelzen der Masse zu treiben, so erhält man eine lebhaft braunrothe Masse, welche an Salzsäure Zink abgibt; da nun das Zinksulfochromit in Salzsäure unlöslich ist, so sehloss ich, dass dabei eine Umsetzung eintritt, die sich durch die Gleichung

$$
\mathrm{ZnCr}_{2} \mathrm{~S}_{4}+\mathrm{Na}_{2} \mathrm{CO}_{3}=\mathrm{Na}_{2} \mathrm{Cr}_{2} \mathrm{~S}_{4}+\mathrm{ZnCO}_{3}
$$

ausdricken lässt. Es gelang aber auf keine Weise, die vermuthete Natriumverbindung von dem Zinkoxyd zu trennen; desshalb wurde versucht, dieselbe auf analoge Weise wie das Zinksulfochromit zu erzeugen, nämlich durch Erhitzen von Natriumearbonat mit Chromhydroxyd und Schwefel im Wasserstoffstrome. Dabei wurde in der That eine rothe Schmelze, die bei der Behandlung mit Wasser ein rothes Pulver zuriickliess, erhalten. Nimmt man an, dass bei diesem Verfahren ein Process sich abspiele, der durch die Gleichung:

$$
\mathrm{Cr}_{2}(\mathrm{OH})_{6}+\mathrm{Na}_{2} \mathrm{CO}_{3}+\mathrm{S}_{6}=\mathrm{Na}_{2} \mathrm{Cr}_{2} \mathrm{~S}_{4}+3 \mathrm{H}_{2} \mathrm{O}+\mathrm{CO}_{2}+2 \mathrm{SO}_{2}
$$

repräsentirt wird, so mïsste man auf 1 Gewichtstheil Chromhydroxyd 0.515 Gewichtstheile Natriumcarbonat und 0.932 Gewichtstheile

1 Wien, Ber. a. k. Akad. d. Wissensch. LXXXI. Bd., Märzheft, p. 531. 
Schwefel nehmen; es zeigt sich aber, dass man dann eine sehr unglcichförmige, stellenweise schwarze Schmelze erhält; soll die Operation gut gelingen, so muss man einen grossen Überschuss an den letzteren beiden Bestandtheilen verwenden; ausserdem erwies sich die Anwendung des Wasserstoffstromes, der den Luftzutritt abhalten sollte, als uiberfliissig, indem der tiberschuissige Schwefel, der bei der Operation verdampft und verbrennt, dessen Rolle übernimmt.

Übergiesst man die erkaltete Schmelze mit Wasser, so geht Natronschwefelleber in Lösung, während ein ziegelrother Niederschlag ungelöst zurückbleibt. Versucht man jedoch, denselben abzufiltriren und mit Wasser auszuwaschen, so bemerkt man, dass, sobald die Natronschwefelleberlösung zum grössten Theile weggewaschen ist, der Niederschlag durch das Filter geht und auf demselben fast gar nichts zuriickbleibt, ferner dass er sich rasch schwärzt, also jedenfalls eine Zersetzung erleidet. Da die Schwärzung an der, der Einwirkung der Luft ausgesetzten Oberfläche des Filtrates beginnt, schloss ich, dass diese Zersetzung durch die oxydirende Wirkung des atmosphärischen Sauerstoffes herbeigeführt werde, und versuchte daher, den Niederschlag in einer sauerstofffreien Atmosphäre, u. zw. im Wasserstoffstrome anszuwaschen; aber einerseits ist der Niederschlag im Wasser so fein vertheilt, dass er sich weder durch Decantation auswaschen, noch durch das dichteste Filter filtriren lässt, anderseits ist die vollständige Ansschliessung des Sauerstoffes der Luft und die Herbeischaffung von luftfreiem Wasser eine so ausserordentlich schwierige, dass es mir nicht gelang, die Schwärzang des Niederschlages zu verhindern.

Behandelt man jedoch die Schmelze mit absolutem Alkohol, so gehen Polysulfide des Natriums in Lösung, und es bleibt ein rother Niederschlag zuriick, der-sich ganz gut abfiltriren, mit absolutem Alkohol auswaschen und dann trocknen lässt, ohne seine Färbung zu verändern, allein der Niederschlag ist, wie man schon mit freiem Auge erkennen kann, nicht homogen, sondern enthält krystallinische Blättchen von Natriumthiosulfat und Natriumsulfat beigemengt; da für letztere ausser Wasser kein Lösungsmittel bekannt ist, so war ich auch auf diese Weise nicht im Stande, das vermeintliche Natriumsulfochromit zu isoliren, 
aber es war aus diesen Versuchen ersichtlich, dass die fragliche Verbindung, sobald sie sich im trockenen Zustande befindet, an der Luft haltbar sei.

Man hat nun längst die Erfahrung gemacht, dass manche in sehr feiner Vertheilung befindliche Niederschläge sich nicht in reinem Wasser, wohl aber in concentrirten Salzlösungen absetzen (z. B. durch Fällung erhaltener Schwefel). Diese Erfahrung suchte ich nun auszunitzen. Es musste dabei aber die Lösung einer Verbindung beniitzt werden, welche erstens anf das vermuthete Natriumsulfochromit nicht chemisch einwirken konnte (am Besten also eine Natriumverbindung), zweitens, sich in Alkohol löst, da man sonst nicht im Stande wäre, den mit derselben ausgewaschenen Niederschlag fiir sich allein im trockenen Zustande zul erhalten.

Eine solche in Alkohol lösliche Natrinmverbindung ist das Ätznatron. Die danit angestellten Versuche gelangen in uberraschender Weise; nicht allein setzt sich der Nieflersehlag in Natronlange rasch $a b$, sondern er lässt sich auch an der luft filtriren und mit derselben auswaschen, olne die geringste Schwärzung zu erleiden. Die Methode, welche ich cinschlug, um diese Verbindung, die sich in der That als

\section{Natriumsulfochromit $\left(\mathrm{Na}_{2} \mathrm{Cr}_{2} \mathrm{~S}_{4}\right)$}

erwies, darzustellen, ist nun folgende:

"Man verreibt $1 \mathrm{Grm}$. bei niederer Temperatur getrocknetes Chromhydroxyd mit $9 \mathrm{Grm}$. wasserfreiem Natriumearbonat and 11 Grm. Sehwefel zu einem homogenen feinen Pulver und füllt mit diesem einen Porzellantiegel bis zu ungefähr ${ }^{4} /$ s seines Inhaltes an, bedeckt denselben mit einem Porzellandeckel und erhitzt auf eine so hohe Temperatur, dass der Schwefel zu verdanpfen und sich am Tiegelrande zu entziunden beginnt, und setzt von diesem Momente an die Erhitzung circa $1 / 4$ Stunde lang fort. Man hört mit dem Erhitzen sehon auf, bevor noch aller überschiussige Schwefel verdampft ist, damit zur Schmelze, so lange sie sich in erhitztem Zustande befindet, der sauerstoff der Luft nicht linzutreten könne.

Die erkaltete Schmelze übergiesst man mit so viel Wasser, als zur Anfiossung der in der Schmelze befindlichen löslichen 
Schwefelverbindungen des Natriums nöthig ist, lässt den ungelöst gebliebenen Niederschlag sich absetzen und wäscht mit einer

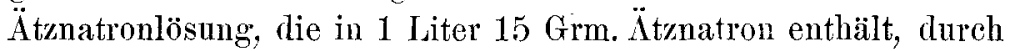
Decantation so lange aus, bis sich in der decantirten Flüssigkeit weder Schwefelnatrium, noch Natriumthiosulfat nachweisen lässt. Sodann wäscht man die Ätznatronlösung, mit welcher der Niederschlag durchtränkt ist, mit starkem Weingeist $\left(96^{0}{ }_{0} \mathrm{Tr}\right.$. $)$ weg, so lange bis die Waschflüssigkeit Curcumapapier nicht mehr bräunt, verdrängt diese schliesslich durch absoluten Alkohol, bringt den Niederschlag auf's Filter, lässt abtropfen und trocknet dann rasch“.

Die so erhaltene Verbindung ist ein dunkel ziegelrothes amorphes Pulver. Im trockenen Zustande erleidet sie beim Liegen an der Luft keine Veränderung. Erlitzt man sie auf höhere Temperatur, so erglüht sic unter Entwicklung ron Schwefeldioxyd und Zuriicklassung eines Gemenges ron Chromsesquioxyd und Natriumsulfat. Dieses Verhalten wurde gleich zar Analyse der Verbindung benitzt, und zwar in folgender Weise: Die Substanz wurde bei $100^{\circ} \mathrm{C}$. bis zur Gewichtsconstanz getrocknet und dann im Sauerstoffstrome erhitzt. Der dabei in Form von Schwefeldioxyd entweichende Schwefel wurde nach der von Zulk ow sky für die Bestimmung des Schwefelgehaltes der Kiese angegebenen Methode ${ }^{1}$ bestimmt und als $\mathrm{BaSO}_{4}$ gewogen. Der aus Chromoxyd und Natriumsulfat bestehende Glihhrickstand wurde gewogen, dann mit Wasser ausgezogen und die gebildete Lösung ron Natriumsulfat von dem ungelöst gebliebenen Chromoxyd abfiltrirt; letzteres wurde gewaschen, getrocknet, geglüht und gewogen. Man findet dann die Menge des Natriumsulfates durch Subtraction. Die Lösung des Natriumsulfates wurde mit Chlorbarium versetzt und das gefällte $\mathrm{BaSO} \mathrm{O}_{4}$ auf gewöhnliche Weise zur Wägung. gebracht, woraus sich die Menge des Schwefels berechnet, welche als Sulfat in dem Gliihrickstande enthalten ist.

0.2445 Grm. Substanz gaben:

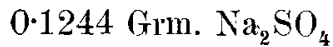

$$
\begin{aligned}
& 0 \cdot 1345 \quad \mathrm{Cr}_{2} \mathrm{O}_{3} \\
& 0.6086, \mathrm{BaSO}_{4} \text { (beim rösten im o als so. } \\
& 0 \cdot 2105 " \mathrm{BaSO}_{4} \text { (als } \mathrm{Na}_{2} \mathrm{sO}_{4} \text { zuriickgeblieben). }
\end{aligned}
$$

1 Zulkowsky, Ber. đ. öst. Ges. z. Förderung d. chem. Industrie, 1881. 
Daraus berechnet sich:

$$
\begin{aligned}
& \mathrm{Na} \ldots \ldots \ldots \ldots \ldots 16 \cdot 48 \% \\
& \mathrm{Cr} \ldots \ldots \ldots \ldots 37 \cdot 75 \\
& \mathrm{~S} \text { (abröstbar) } \ldots \ldots 34 \cdot 19 \\
& \mathrm{~S} \text { (nicht abröstbar) } 11.82 \\
& \frac{100 \cdot 24 \%}{0}
\end{aligned}
$$

$$
\begin{aligned}
& \frac{\begin{array}{c}
\text { Die Formel } \mathrm{Na}_{2} \mathrm{Cr}_{2} \mathrm{~S}_{4} \\
\text { verlangt }
\end{array}}{16 \cdot 49 \%} \\
& 37.63 \\
& 34 \cdot 41 \\
& \frac{11 \cdot 47}{100 \cdot 00^{0} / 0^{\circ}}
\end{aligned}
$$

Diese Verbindung ist also in der That Natriumsulfochromit. Zu gleicher Zeit ergibt sich daraus, dass beim Erhitzen derselben im Sauerstoffstrome der durch die Gleichung

$$
\mathrm{Na}_{2} \mathrm{Cr}_{2} \mathrm{~S}_{4}+\mathrm{O}_{13}=\mathrm{Na}_{2} \mathrm{SO}_{4}+\mathrm{Cr}_{2} \mathrm{O}_{3}+3 \mathrm{SO}_{2}
$$

ausdriickbare Process vor sich geht.

Diese Verbindung ist in Wasser unlöslich; rülurt man sie in demselben auf, so geräth sie in einen Zustand ausserordentlich feiner Vertheilung, so dass sie sich, wie schon erwälnt, nicht abfiltriren lässt und beginnt sich dabei zu schwär\%en. Da auch noch bei der Behandlung des Niederschlages mit Wasser, welches dureh Auskochen vermeintlich ron Luft befreit war, immer Bräunung desselben eintrat, so glaubte ich anfangs, dass die Verbindung dureh Wasser selbst zersetzbar sei. Diese Bräunung riihrt aber nur davon her, dass man durch blosses Auskochen nicht vollkommen sauerstofffreies Wasser herstellen kann. Um mir ein solches zu verschaffen, versetzte ich ausgekochtes Wasser mit Eisenvitriollösung und Kalilauge und liess das Ganze in einem Wasserstoffstrome, der dureh Eisenhydroxydul ebenfalls von etwas beigemengtem Sanerstoffe befreit wurde, einige Zeit stehen. Sodann wurde das auf diese Art seines Sanerstoffes beraubte Wasser im Wasserstoffstrome in eine ebenfalls von Wasserstoff durchströmte Vorlage, welche das Natriumsulfochromit enthielt, iiberdestillirt. Die Vorlage wurde dann luftdicht verschlossen. Der Niederschlag erlitt nicht die geringste Farbenveränderung, setzte sich aber erst nach monatelangem, ruhigem Stehen vollkommen ab.

Vertheilt man jedoch das Natrinmsulfochromit in einer Lösung von Ätznatron von oben angegebener Concentratiơn, so setzt es sich ziemlich rasch ab und erleidet auch bei wochen- 
langem Stehen an der Luft, sowie anch beim Durchsaugen eines Luftstromes durch die Flüssigkeit keine Schwärzung. Um die Wirkung des Ätznatron zu erklären, glaubte ich annehmen zu dürfen, dass diese durch eine Schwärzung sich kundgebende Zersetzung von der Einwirkung der Kohlensäure der Luft herrïhre, dass die Kohlensäure zuerst vom Ätznatron absorbirt werde, und letzteres so das Natriumsulfochromit vor der Zersetzung schïtze. Allein diese Schwärzıng tritt auch auf, wenn man durch das Wasser, in welchem das Natriunsulfochromit vertheilt ist, einen vollkommen kohlensäurefreien Luftstrom leitet. Der dabei entstehende schwarze Niederschlag befindet sich ebenfalls in sehr feiner Vertheilung und lässt sich erst abfiltriren, wenn die Flüssigkeit, in welcher er suspendirt ist, durch Eindampfen stark concentrirt wird; er erwies sich als Chromsesquisulfid. Im Filtrat liessen sich Polysulfide des Natriums, Natriumthiosulfat und Natriumhydroxyd, also die Oxydationsproducte des Natriummonosulfides nachweisen, woraus ersichtlich ist, dass die eintretende Zersetzung eine Oxydationserscheinung ist. Die Ursache, warum die Ätznatronlösung diese Oxydation verhindert, ist mir vollständig unerklärlich; man mïsste nur annehmen, dass erst die äusserst feine Vertheilung des Natriumsulfochromites im Wasser diese leichte Oxydirbarkeit hervorrufe, und dass die Natronlauge diese feine Vertheilung (wie dies ja auch aus der raschen Absetzbarkeit des Niederschlages in derselben herrorgeht) verhindere. (Ein ähnliches Verhalten zeigt der Eisenkies, der sich in feuchtem Zustande nur in sehr feiner Vertheilung an der Luft von selbst oxydirt, so dass ein Thonschiefer, der Eisenkiestheilchen von solcher Grösse enthält, dass sie schon mit freiem Auge wahrgenommen werden können, sich zur Alaungewinnung, oder zur Darstellung von Vitriolstein sehr schlecht oder gar nicht eignet.)

Concentrirte Salzsäure greift das Natriumsulfochromit nicht, verdünnte Salzsäure, sowie verdünnte Schwefelsäure beim Erhitzen etwas an, wobei eine geringe Schwefelwasserstoffentwicklung bemerkbar ist; wahrscheinlich sind es aber eigentlich nur die durch den Gehalt der Säuren an freiem Sauerstoff erzeugten Zersetzungsproducte desselben, welche von der Säure gelöst werden. In concentrirter Salpetersäure und Königswasser hingegen ist es schon in der Kälte, in verdünnter Salpetersäure 
beim Erwärmen unter Bildung der Sulfate des Chroms und Natriums leicht löslich. Concentrirte Schwefelsäure hat in der Kälte fast keine Einwirkung, beim Erhitzen jedoch tritt znerst eine Schwärzung, sodann unter Schwefeldioxydentwicklung und Schwefelabscheidung eine vollständige Lösung der Verbindung ein, es entsteht dabei eine Natrinmsulfat und Chromsulfat enthaltende grüne Lösung.

Nun versnchte ich auf ganz dieselbe Weise das Kaliumstulfochromit darzustellen, indem ich das Natriumcarbonat durch eine äquivalente Menge Kaliumcarbonat ersetzte. Merkwiirdigerweise verhält sich aber das. Kaliumcarbonat ganz anders; man erhält, selbst wenn man einen noch viel grösseren Überschuss an Kaliumearbonat und Schwefel nimmt, als dies bei der Darstellung des Natriumsulfochromites geschehen, eine schwarze Schmelze, welche an Wasser die Polysulfide des Kaliums und Kaliumthiosulfat unter Zurücklassung eires schwarzen Niederschlages von krystallinischem Aussehen, der sich sehr gut abfiltriren und auswasschen lässt, abgibt. Der bei $100^{\circ}$ (\% getrocknete Niederschlag erwies sich bei der Analyse als Chromsesquisultid, welches nur geringe Mengen von Kalium $(0 \cdot 6 \%)$ enthielt. In der Meinung, dass beim Auswaschen des Niederschlages mit Wasser ein analoger Vorgang stattfinde, wie beim Natriumsulfochromit, versuchte ich auf dieselbe Weise, wie dies bei letzterem geschehen, die vermuthete Zersetzung durch Auswaschen des Niederschlages mit Kalilauge und Verdrängen dieser Waschflüssigkeit durch Alkohol die vermeintliche Kaliumverbindung zu isoliren, allein auch auf diese Weise erhielt ich ein Product, das nur $1 \cdot 3 \%$ Kalium enthielt, im Übrigen aber die Zusammensetzung des Chromsesquisulfides zeigte. Auch auf andere Art, wie z. B. durch Ersatz des Kaliumcarbonates durch Kaliumhydroxyd oder durch Eintragen von Chromsesquisulfid in schmelzende Kalischwefelleber konnte jch eine der Formel $\mathrm{K}_{2} \mathrm{Cr}_{2} \mathrm{~S}_{4}$ entsprechende Verbindung nicht erhalten. Die Bildung eines solchen kalihältigen Chromsulfides aber beobachtete sehon Schafařik' beim Schmelzen von Kaliumchromat mit Schwefelleber.

I Schafằik, Wien, Ber, d. k. Akarl. d. Wissensch. 47, 2, p. 25;. 
Da das Natriumsulfochromit mit Metallsalylösungen behandelt die entsprechenden Metallsulfochromite liefert, so konnte es als Ausgangspunkt für die Darstellung derselben beniitzt werden. $\mathrm{Zu}$ diesem $\mathrm{Zwecke}$ wurde in folgender Weise verfahren: „Aus der concentrirten Lösung des entsprechenden Metallchlorides (bei der Darstellung der Silber- und Bleiverbindung wurden die Nitrate verwendet) wurde zuerst durch anlaaltendes Kochen die Luft so gut wie möglich ausgetrieben, um die Oxydation des Natriumsulfochromites, welches darauf in einen Überschuss derselben eingetragen wurde, und dadurch die Bildung secundärer Producte zu verhindern; nach etwa einstündigem Erhitzen wurde der Niederschlag abfiltrirt, mit heissem Wasser gewaschen und getrocknet. Ich erhielt auf diese Weise die Sulfochromite des Silbers, Bleies, Kupfers, Kadmiums, Zinns, Kobalts, Nickels, Eisens, Mangans und Zinks.

Die Eigenschaften dieser verschiedenen Sulfochromite weichen so wenig von einander ab, dass es angezeigt erscheint, dieselben in Einem zu beschreiben. (Die auf nassem Wege erzeugten Súlfochromite des Eisens, Mangans und Zinks zeigen mit Ausnahme der dunkleren Farbe dieselben Eigenschaften, wie die friiher von mir auf trockenem Wege dargestellten und in oben citirter Abhandlung beschriebenen Verbindungen.)

Die iubrigen Sulfochromite sind sämmtlich schwarze, oder grauschwarze, pulverförmige, in Wasser unlösliche Substanzen. Von Salzsäure werden dieselben nicht angegriffen, hingegen lösen sie sich in Salpetersäure unter Oxydation leicht auf, die entsprechenden Matallsalze bildend. Das Stannosulfochromit hinterlässt dabei Metazinnsäure, das Bleisulfochromit Bleisulfat. Auch in Königswasser sind alle leicht löslich, das Silbersulfochromit natürlich unter Zurïcklassung von Chlorsilber.

Beim Erhitzen an der Luft verglimmen die Sulfochromite unter Entwicklung von Schwefeldioxyd. Der Gluhriickstand besteht bei den Sulfochromiten vom Silber, Kupfer, Kadmium, Kobalt, Nickel und Mangan aus Chromoxyd und den Sulfaten der entsprechenden Metalle, bei dem Eisen- und Stannosulfochromit aus Chromoxyd und Eisenoxyd, respective Zinnoxyd; Blei- und Zinksulfochromit hinterlassen die Chromite diese Metalle. 


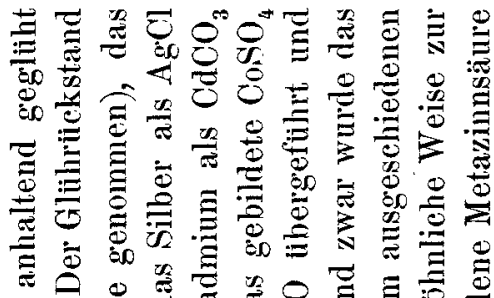

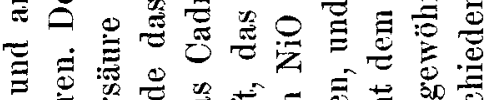

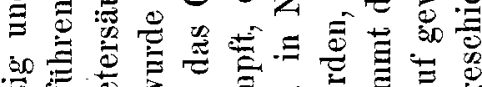

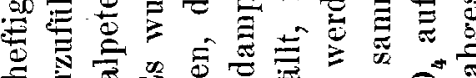

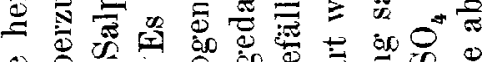
谣:

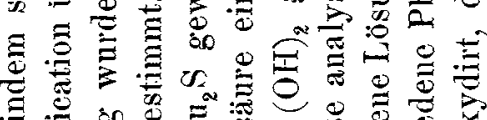

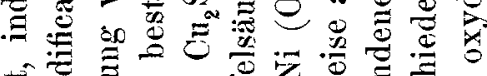

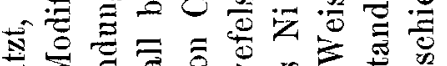

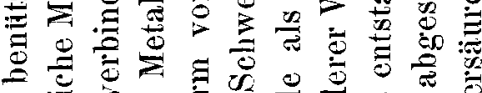
菏

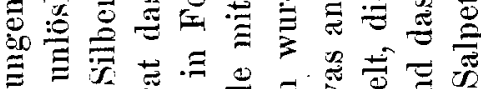

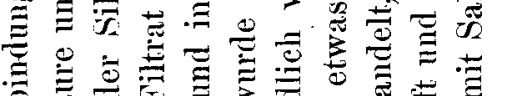

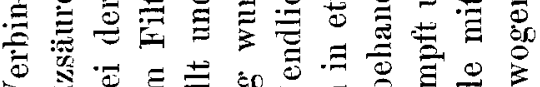

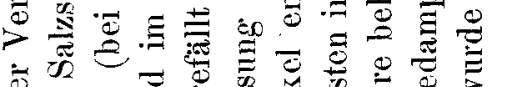

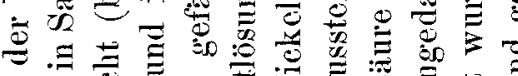

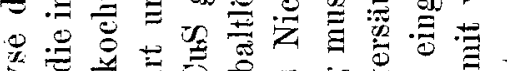

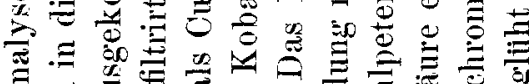

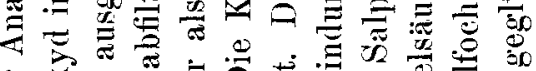

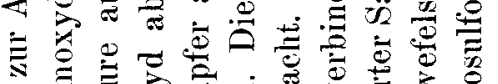

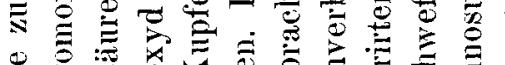

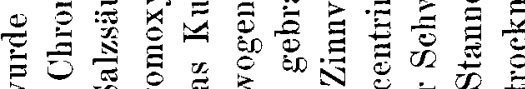

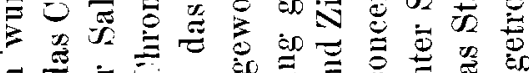
ह

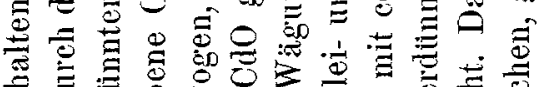

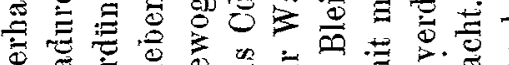

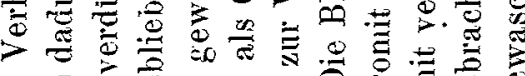

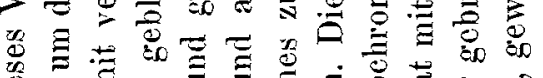

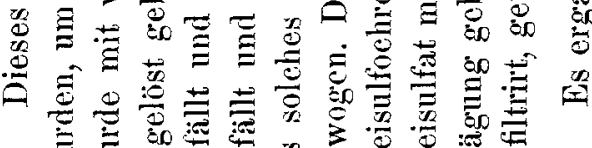

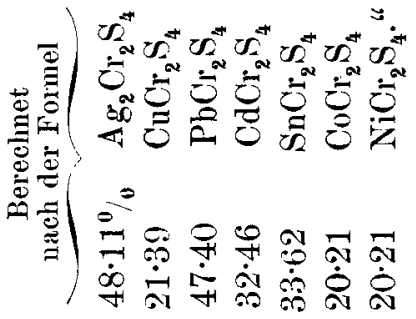

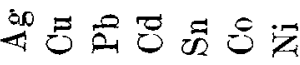

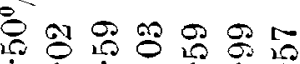

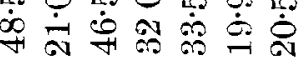
递

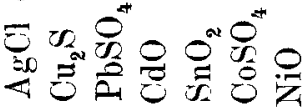

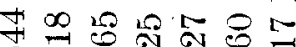

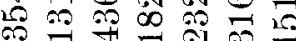

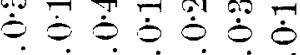

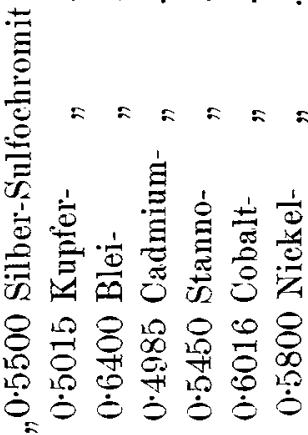


Die Sulfochromite des Bariums, Stronziums und Calciums lassen sich aus dem Natriumsulfochromit durch doppelte Zersetzung nicht darstellen, indem diese Umsetzung nur sehr unvollständig und langsam erfolgt, aus welchem Grunde auch das Natriumsulfochromit Gelegenheit findet, sich zu oxydiren und die früher beschriebenen Zersetzungsproducte zu liefern.

Die Existenz der beschriebenen Sulfochromite zeigt, dass das Chromsesquisulfid den Sulfiden vieler Metalle gegenüber sich gerade so verhält, wie das Chromoxyd zu den Oxyden derselben. Da nun das Aluminiumoxyd dem Chromoxyd in vielen Stiucken sich ähnlich verhält, so liegt die Frage nahe, ob diese Analogie auch zwischen dem Aluminiumsulfid und dem Chromsulfid bestehe, eine Frage, mit deren Lösung ich noch beschäftigt bin, und über welche ich nächstens berichten zu köninen hoffe. 